\title{
EL CUIDADO PERCIBIDO DURANTE EL PROCESO DE PARTO: UNA MIRADA DESDE LAS MADRES
}

\author{
Paulina Bravo V. ${ }^{a}$, Claudia Uribe T. ${ }^{b}$, Aixa Contreras M. ${ }^{b}$ \\ Departamento de Salud de la Mujer, Escuela de Enfermería, Pontificia Universidad \\ Católica de Chile.
}

${ }^{a}$ Enfermera Universitaria, ${ }^{b}$ Enfermera Matrona.

\section{RESUMEN}

Antecedentes: La humanización de la atención de salud constituye un foco de interés social, transformándose en una estrategia que permite incrementar el bienestar de los usuarios. En salud de la mujer, el cuidado humanizado, tiene especial importancia en la atención del parto. En él, mujer e hijo/a experimentan una situación de vulnerabilidad y el buen cuidado recibido, constituye un elemento clave en la percepción de satisfacción y bienestar referido por la mujer. Objetivo: Responder la pregunta: ¿Cuál es la percepción del buen cuidado que tienen las mujeres en situación de parto? Método: Se realizó un estudio bibliográfico, considerando los años 1999 hasta el 2006, obteniendo 20 artículos de investigación cualitativa y cuantitativa, de primera fuente, a texto completo en inglés. Resultados: El análisis develó la percepción del cuidado, que tienen las protagonistas del proceso, sustentado en dos componentes principales. El componente técnico, se refiere a la experticia que la mujer reconoce, en los procedimientos efectuados por los profesionales. El componente relacional, al que asignan el valor principal, indica que el uso de palabras suaves, el aporte de información, la presencia de un familiar, el apoyo y respaldo de las madres, son considerados elementos fundamentales para que la mujer en situación de parto, pueda vivirlo satisfactoriamente, traduciéndose el buen cuidado, en eje de bienestar y valoración personal, permitiéndoles sentirse partícipes de su experiencia. Conclusión: Lo anterior, permite afirmar que los objetivos profesionales deben considerar como eje organizador del cuidado, el desarrollo de habilidades interpersonales, sin olvidar la permanente actualización del componente técnico del cuidado.

\section{PALABRAS CLAVE: Proceso de parto, percepción, cuidado}

\section{SUMMARY}

Background: Health attention humanization is been an interesting social focus and it could be a strategy that can grow up user well-being. In women's health, humanized care has a special relevance in childbirth. In it women and daughter/son live a vulnerability situation and well-care is a key element in satisfaction perception and wellbeing for women. Objective: To respond the answer: which is the perception of well-care who has women living childbirth? Method: It had been make a literature review since 1999 to 2006, obtaining 20 qualitative and quantitative studies at first author, full text in English. Results: Data analysis demonstrated that care perception that has women in childbirth is sustained in two principal components. Technique component refer to expertise that women identify in the professional procedures. The relational component, with the biggest value, affirm that soft words, give information, family presence and support are considerer fundamental elements for women satisfaction in childbirth- and it could translate well-care like a central point 
in well-being and make that women can participate in their experience. Conclusion: Professional goals have to considerer interpersonal skills like principal element of care, but we cannot forget continuous actualization of technical component of care.

\section{KEY WORDS: Childbirth, perception, care}

\section{INTRODUCCIÓN}

El proceso del parto representa una de las experiencias más paradójicas que vive la mujer. Por un lado crea vida, constituyendo para algunas lo mejor que pudo pasar tras el embarazo (1), pero a la vez puede ser el evento más doloroso que experimenta, implicando una experiencia psicosocial profunda, que pone a prueba su feminidad y competencias personales $(2,3)$, limitando sus habilidades funcionales, principalmente en la disminución del control que ella puede mantener frente a su propia fisiología (3). Por este hecho, la idea de que la mujer necesita ser acompañada durante este proceso no es nueva, incluso antes de que se instaurara la matronería y enfermería, era habitual encontrar a mujeres que se dedicaban a acompañar a otras durante este proceso (4).

Una vez que las matronas y enfermeras se posicionaron como profesión, surge también el apoyo durante el proceso de parto, como una de las actividades a realizar en pro de la mujer. Pese a ello, no existe consenso respecto a qué elementos del cuidados deben brindarse para que la mujer perciba el "apoyo" (5).

Cabe señalar, que las energías de los profesionales de salud involucrados en el proceso de parto, se han centrado durante los últimos dos siglos en "tecnocratizar" la atención brindada (4), enfatizando al medio físico como principal factor, especialmente en la disminución de las tasas de infecciones (6), lo que sin duda ha sido efectivo, lográndose una disminución en las tasas de mortalidad materna e infantil (7). Esta priorización, que concuerda con el enfoque biomédico actualmente predominante en los modelos de atención de salud, ha subvalorado y en muchos casos ignorado el componente místico y simbólico que acompaña esta vivencia, descuidando con ello el llamado que las personas hacen a los agentes sanitarios, para que aumenten la calidad del cuidado interpersonal (6).

Sin embargo, a partir de algunas décadas atrás, numerosas iniciativas y políticas públicas internacionales y recientemente nacionales, han intentando dar un vuelco a esta tendencia, tratando de humanizar la atención en salud desde el primer momento de cada persona, es decir, desde el nacimiento (8).
En el ámbito del cuidado profesional en la situación de parto, la humanización corresponde principalmente, desde la perspectiva de las usuarias, al ámbito del bienestar personal, relacionado con la calidad de la atención brindada por los profesionales (9).

Lundgren y Dahlberg (10) plantean que la relación que se establece entre la mujer y la matrona, es el factor clave de un buen apoyo durante el proceso de parto. La satisfacción con el proceso se encuentra referida, a su vez, con el bienestar de la madre y del niño, tendiendo a relacionarse incluso con la calidad del vínculo que se establecerá entre ellos (11).

Adicionalmente, investigadores nacionales a través del proyecto FONIS SA05I20050 (12) han construido una herramienta de valoración, que permite objetivar el bienestar materno en el proceso del parto, relevando con ello el equilibrio que debe existir entre el bienestar de la madre, del hijo y la progresión adecuada y natural del proceso de parto, en un entorno acorde con sus necesidades, lo que está fuertemente vinculado al trato o cuidado que reciben la madre y el hijo desde el equipo de salud.

Siguiendo esta línea, surge la necesidad de identificar la participación que tiene el cuidado percibido en el bienestar materno, a través de la siguiente pregunta: ¿Cuál es la percepción que tienen del buen cuidado, las mujeres que viven el proceso de parto?

El objetivo general que pretende este estudio bibliográfico es conocer la percepción del buen cuidado de las mujeres en proceso de parto, y los objetivos específicos son develar el bienestar o satisfacción que experimentan las mujeres, respecto al cuidado recibido en el proceso de parto y comprender cuáles son los componentes que determinan la satisfacción durante el proceso de parto.

\section{MATERIAL Y MÉTODO}

La búsqueda bibliográfica se realizó en las bases de datos PROQUEST, SCOPUS, ELSEVIER, EBM, GOOGLE SCHOLAR y CINHAL, durante los meses de agosto y diciembre del año 2006. Los resultados se limitaron a artículos de primera fuente, 
en inglés, publicados desde el año 1999 en adelante, cuyo texto completo se obtuviera de manera gratuita. Las palabras clave utilizadas fueron childbirth, childbearing, care, perception, wellbeing, satisfaction. Se seleccionaron veinte artículos de investigación; catorce de ellos reportan metodología cualitativa, los cuales fueron analizados a través la guía creada por Rivera (13). Tres explicitan un diseño fenomenológico, ocho análisis de contenido, dos etnometodologías (una etnografía) y una teoría fundada. Las principales limitaciones apuntan hacia la omisión de la selección o saturación muestral y de los aspectos éticos que los respaldan. Los seis estudios restantes pertenecen a la metodología cuantitativa. Sus diseños corresponden a un ensayo clínico randomizado controlado, y cinco analíticos de corte transversal. Las principales limitaciones se centran en la omisión del reporte de la validez de los instrumentos a utilizar en la fase de recolección de la información.

\section{RESULTADOS}

Las participantes de los estudios son mujeres entre 15 a 45 años, de los distintos estratos socioeconómicos y educacionales; provenientes de culturas orientales, oceánicas y anglosajonas. Incluyen a mujeres con una primera experiencia de parto o con más de una. La recolección de la información se realizó durante la etapa postparto o hasta 36 meses posterior al nacimiento. Los estudios se desarrollaron en ambos sistemas de atención de salud, abarcando el área urbana y rural.

\section{Percepción del cuidado desde las perspectivas} cualitativas y cuantitativas.

Cuidado técnico versus cuidado relacional: A través del análisis, se han desprendido temáticas provenientes de unidades de significados o codificaciones, según el diseño metodológico desde el que provenían. Para fines de organización y entendimiento se diseñó un esquema que pretende explicar los hallazgos (Figura 1). En base al esquema, el buen cuidado aparece como temática principal en la atención profesional hacia la mujer en el proceso de dar a luz; de él se desprenden dos temas centrales que lo sustentan y se interrelacionan. Cada uno de ellos se compone de unidades que significan esta vivencia desde la perspectiva de las madres. Como se aprecia, el componente relacional aparece como categoría principal, reuniendo la mayoría de la información. Sin embargo, pese a lo anterior durante una observación de campo se identificó, que las enfermeras brindan escaso tiempo de apoyo a las madres, entendido como la promoción del confort, el apoyo emocional, la entrega de información y la abogacía; reportando que los profesionales estudiados ocuparían, en su jornada laboral, sólo el $12,4 \%$ del tiempo, siendo inexcusable por medio de la carga laboral, la ausencia de un cuidado más integral (14). Este hecho es preocupante, puesto que al comparar a un grupo de mujeres que permanecen en soledad durante el proceso de parto, versus las que están acompañadas por algún familiar, aparece una diferencia estadísticamente significativa, pues las primeras, prefieren ser acompañadas por un profesional de la salud durante este proceso, por sobre la compañía familiar ( $p=0,0001)$. Lo que sin duda es un llamado a los profesionales, respecto a la entrega oportuna de acompañamiento hacia las mujeres, ya sea desde la familia, o a través del equipo de salud (15). Pues la satisfacción de las mujeres durante el proceso de parto está determinada mayoritariamente $(53 \%)$ por el componente relacional desarrollado por las enfermeras (16).

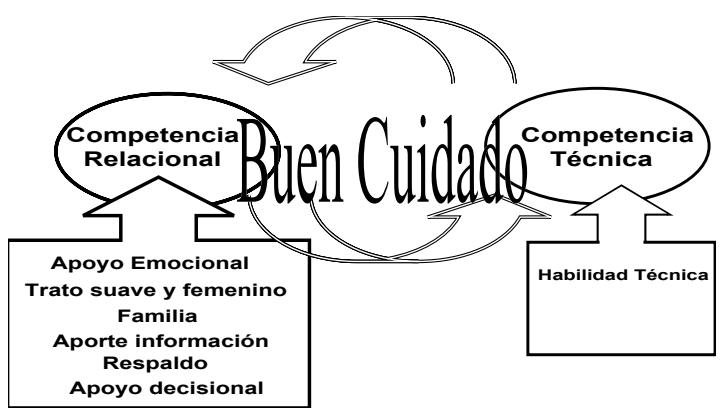

Figura 1. Esquema de la estructura de los hallazgos provenientes de los artículos de investigación cualitativa.

\section{Percepción del buen cuidado y la competencia técnica.}

Esta dimensión del cuidado, surge escasamente desde la evidencia cualitativa, haciendo referencia a las competencias técnicas-procedimentales de los profesionales involucrados en el proceso del parto. Las mujeres son sometidas a innumerables procedimientos durante el parto, principalmente en instituciones docente asistenciales, donde son realizados en forma repetitiva y centrados en la progresión del proceso (7), los que podrían facilitar el trabajo de parto, siendo considerados por las mujeres como una ayuda técnica de parte del profesional (17). Si no son realizados en forma delicada, pueden ser percibidos como malestar físico y emocional (7), además, pueden ser considerados como situaciones riesgosas, tanto para la madre como para el hijo; frente a ello las madres se sienten más seguras si las enfermeras demuestran habilidades 
en los procedimientos y lo hacen profesionalmente (18): "Most important of all, their knowledge of technological equipments in the hospital and their professional technical skills gave us the greatest reassurance" (18).

\section{Percepción del buen cuidado y la competencia relacional.}

a) Apoyo emocional profesional. La satisfacción durante este proceso depende fuertemente del nivel de interacción con el equipo de salud $(19,20)$. Esto hace distinguir a dos tipos de enfermeras, las que se aproximan solo desde lo físico y las que lo hacen además desde lo relacional, enfoque centrado en la persona, lo que es evidentemente reconocido por las mujeres que viven el proceso $(7,21)$ : "For me it was extremely valuable that she understood and could help me to set goals of how I would do" (21). Este apoyo emocional incluye empatía, compasión y palabras suaves, lo que alienta a la madre (18) y la hace partícipe del proceso $(22,17)$, haciendo que la satisfacción con el proceso de dar a luz se incremente (16). Además, el apoyo y la confidencialidad que las madres identifiquen en el personal de salud, puede determinar que ellas recomienden el servicio de la maternidad donde han sido atendidas, constituyéndose en un determinante en la elección del servicio de salud donde se atenderán (23), lo que puede ser entendido como un indicador de satisfacción con el servicio brindado (19). Frente a ello, mantener un cuidado continuo es fundamental, en la medida que es agradable para el $91 \%$ de las madres que lo reciben, permitiéndoles sentirse seguras durante el proceso de parto, sintiendo que conocen a las matronas a cargo de su cuidado (24). Esto tiene una importante repercusión en sentir que la situación está bajo su control (25).

b) Trato profesional suave y femenino. Las enfermeras durante el parto, pueden ser percibidas por las madres como confortables, en la medida que son suaves en el trato, acarician, ayudan, sonríen y brindan un cuidado satisfactorio $(18,16)$; lo anterior puede relacionarse, con las preferencias maternas por ser cuidadas por personal femenino, quienes podrían desarrollar de manera más efectiva los rasgos antes señalados $(7,20)$, explicando que aproximadamente el $90 \%$ de las mujeres participantes en un estudio, elegirían ser atendidas por una mujer, si tuvieran la oportunidad de hacerlo (15): "...She was very patient, kind, and had a warm voice and smile" (7). Adicionalmente el uso de palabras de elogio, ayuda a las mujeres a aumentar su autoestima y la satisfacción durante el proceso vivido $(20,22)$. No obstante, durante una observación de campo, se develó que el contacto táctil del personal de salud hacia la madre se limita a los procedimientos que deban realizarle, lo que se traduce en la tecnificación de las caricias (7).

c) Facilitar la presencia de un familiar. Durante el proceso de parto, las mujeres reportan la necesidad de recibir amor y apoyo desde la familia, fundamentalmente desde la madre y la pareja, pues el cuidado otorgado por ellos proviene desde el corazón (7): "I begged them to let my mum in with me. She would touch me, hold my hand, support me... It's very hard to feel lonely in labour" (7). En una de las investigaciones se develó que, pese a que las mujeres no tuvieron la opción de permanecer con sus esposos durante el parto, consideraron que su presencia es necesaria (20). Facilitar la cercanía de los seres significativos es un determinante para la recomendación de la maternidad por parte de la madre $(O R=0,78)$, lo que se relaciona directamente con la satisfacción con la atención (19). Por el contrario el $15,9 \%$ de las primíparas participantes, sintieron disminuido el control que podían mantener de la situación de parto, al no encontrarse acompañadas, lo que podría influir en la satisfacción referida en el proceso (25).

d) Aporte de información efectiva. El miedo aparece en este proceso como un sentimiento intenso de difícil exteriorización, abarcando desde miedo al dolor, hasta cercanos sentimientos de muerte $(21,26)$. Frente a esto se revela que la información ayuda a las mujeres a orientarse a la realidad y aliviar sus temores, permitiéndoles adquirir seguridad tanto en el proceso del parto, como en el personal que otorga el cuidado (18): "They moniroted how I was doing every few minutes and let me know what they had observed... I really could feel secure" (18). Aportar adecuada información a las mujeres durante el proceso de parto, puede predecir y aumentar el nivel de control del proceso $(p=0,000)(24)$. Además es un determinante de la satisfacción, en la medida que durante la interacción informativa efectiva y centrada en la madre sienten escuchadas sus preocupaciones y aclaradas sus dudas $(19,20)$.

e) Respaldo profesional de la madre, la abogacía. Las mujeres agradecen el apoyo a sus decisiones cuando sienten que pueden realizarlas, identificando este respaldo como una actividad ayudadora del cuidado $(18,27)$; esto es, una de las expectativas positivas, referidas por las madres, respecto al proceso de parto (26). Sin embargo, cuando las mujeres perciben que no pueden hacerlo, relatan que son las enfermeras quienes deben abogar por sus intereses, ya sea a nivel físico, en el manejo del dolor, como en situaciones interpersonales, que pueden entorpecer el proceso $(26,17,16)$ : "Basically, I just wanted that opportunity to like choose the way my labour was going to progress but if I did need to 
make choices like about pain relief that I would be supported by the midwives and my doctor" (26).

f) Favorecer la participación materna en la toma de decisiones y el autocontrol. Durante el proceso de parto las mujeres ven menoscabado el control sobre su fisiología (3). Sobretodo debido al dolor que implica dar a luz (28). Ante esto las madres reportan que el control que pueden mantener durante el proceso es importante $(1,29)$, pues aumenta la satisfacción con el manejo del dolor $(p=0,002)$ (28), incrementando la percepción de bienestar (25). Durante una investigación se determinó que el autocontrol que las mujeres mantienen durante el proceso de parto, es la única variable estadísticamente significativa relacionada con la satisfacción durante este proceso $(p=0,005)$ (11). Permitiéndoles de esta manera participar activamente en la toma de decisiones durante el parto, en la medida que ellas tienen el control de la situación $(18,19)$. Además las mujeres esperan que sus cuidadores respeten sus deseos de mantenerse bajo el control de la situación de parto. Sin embargo, cuando sienten que el control ha desaparecido, surge la rabia como sentimiento predominante, pues se sienten mal cuidadas y atendidas por un equipo incompetente (30). No obstante solo el $22 \%$ de las mujeres participantes en una investigación, sintieron haber mantenido el control durante el proceso de parto, dato que representa un importante cuestionamiento a los agentes sanitarios (25): "They didn't involve me in my care, and they didn't let me know what was going on. They gave the appearance of being incompetent, and they gave the appearance of being uncaring... It just really made me angry on a lot of different levels" (30).

\section{DISCUSIÓN}

La satisfacción con el cuidado durante el proceso de parto, es concebida por las mujeres a través del desarrollo de dos componentes; uno técnico, menos fuerte, pero no por esto menos importante y el componente puramente relacional. La competencia técnica se relaciona con la percepción del buen cuidado, en la medida que las mujeres le asignan un valor a los procedimientos que tienden a conducir en forma adecuada la progresión del proceso. No obstante, estos deben ser realizados en forma cuidadosa y delicada.

El componente más fuerte de la percepción del buen cuidado corresponde a la competencia relacional, que permite que las madres se sientan escuchadas, miradas, valoradas y complacidas en sus deseos e inquietudes. Esta competencia se sustenta principalmente en el apoyo emocional percibido por las mujeres, desde los profesionales de salud, y por el empoderamiento de ellas a lo largo del proceso de parto.

La entrega del componente relacional efectivo y centrado en el favorecimiento de la mantención del control de las mujeres en su propia situación de parto, ha demostrado un aumento significativo en el nivel de satisfacción de la madre durante la atención recibida en el proceso (11). Esto promueve el posicionamiento activo de ellas en el proceso de parto, lo que les permite adquirir seguridad (23) y mantener control sobre el propio dolor (28).

Frente a ello, los profesionales de la salud, en contacto directo con las mujeres en proceso de parto, están llamados a procurar el fortalecimiento de las interacciones relacionales de calidad y satisfaciendo las necesidades maternas respecto a las mismas (16).

Pareciera ser que lo anterior, puede traducirse a la práctica solo a través de un cambio en el modelo de atención de parto que impera en la actualidad, focalizado en lo cuantificable y tangible. Este nuevo modelo de cuidado debe centrarse en la relevancia de los requerimientos de la mujer y su hijo/a, involucrándola a lo largo del proceso que vive, sin olvidar que ella tiene capacidades que le permiten participar y controlar su proceso activamente.

La enfermería como profesión de salud que se encuentra más cercana a los usuarios y que además le compete el rol de gestor del cuidado (31), debe velar por este cambio desde lo puramente positivista y biomédico, hacia la humanización de la atención del proceso de parto, apuntando hacia la horizontalidad de la atención, donde usuaria y profesional se encuentran en un mismo nivel y se percibe igualdad, lo que permite que la mujer se sienta tratada dignamente, de un modo personalizado y humanizado.

Es importante sugerir que, acudir a los trabajadores de la salud involucrados en el proceso de parto, puede ser de utilidad, al develar cuál es la percepción que ellos tienen del cuidado brindado, tendiendo con ello a la humanización del proceso de parto, al enfocarse las energías de los distintos actores en el proceso, hacia el mismo objetivo, es decir, lograr que la mujer viva de manera satisfactoria el inolvidable proceso de dar a luz.

\section{BIBLIOGRAFÍA}

1. Callister LC, Vehvilainen-Julkunen K, Lauri S. Giving birth: Perceptions of finnish chilfbearing women. MCM 2001;26(1):28-32.

2. Callister LC, Khalaf I, Semenic S, Kartchner R, Vehvi- 
lainen-Julkunen K. The Pain of Childbirth: Perceptions of Culturally diverse women. Pain Management Nursing 2003;4(4):145-154.

3. Perla L. Patient compliance and satisfaction with nursing care during delivery and recovery. Journal of Nursing Care and Quality 2002;16(2): 60-66.

4. Papagni K, Buckner E. Doula support and attitudes of intrapartum nurses: a qualitative study from the patient's perspectiva. Journal of Perinatal Education 2006;15(1): 11-18.

5. Sauls DJ. The labor support questionnaire: Development and psychometric analysis. Journal of Nursing Measurement 2004;12(2):123-132.

6. El-Nemer A, Downe S, Small N. 'She would help me from the heart': An ethnography of Egyptian women in labour. Social Science and Medicine 2006;62(1):8192.

7. Martell LK. Postpartum women's perceptions of the hospital environment. J Obstet Gynecol Neonatal Nurs 2003;32(4):478-85.

8. Consejo Asesor Presidencial para la Reforma de Políticas de Infancia. Propuestas del Consejo Asesor Presidencial para la Reforma de Políticas de Infancia. 2006

9. Jackson JL, Chamberlin J, Kroenke K. Predictors of patient satisfaction. Social Science and Medicine 2001;52(4): 609-620.

10. Lundgren I, Dahlberg K. Midwives 'experience of the encounter with women and their pain during childbirth. Midwifery 2002;18: 155-164.

11. Goodman P, Mackey M, Tavakoli A. Factors related to childbirth satisfaction: issues and innovations in nursing practice. Journal of Advanced Nursing 2004;46(2):212-219.

12. Uribe C, Contreras A, Rivera MS, Contreras O, Cornejo $\mathrm{M}$, Villarroel $\mathrm{L}$. Construyendo una herramienta de evaluación de bienestar materno durante el proceso de parto desde una perspectiva cuali-cuantitativa FONIS SA05I20050. 2006.

13. Rivera MS. Guía para la evaluación crítica de investigación cualitativa. Manuscrito no publicado, Pontificia Universidad Católica, Chile.

14. Gale J, Fothergill-Bourbonnais F, Chamberlain M. Nursing support during childbirth. MCN The American Journal of Maternal Child Nursing 2001;26(5):264271.

15. Mosallam M, Rizk DEE, Thomas L, Ezimokhai M. Women's attitudes towards psychosocial support in labour in United Arab Emirates. Archives of Gynecology and Obstetrics 2004;269(3):181-187.

16. Tumblin A, Simkin P. Pregnant women's perceptions of their nurse's role during labor and delivery. Birth $2001 ; 28(1): 52-56$.
17. Melender HL. What Constitutes a Good Childbirth? A Qualitative Study. Journal of Midwifery \& Women's Health 2006;51(5):331-339.

18. Chen $\mathrm{CH}$, WaNg SY, Chang MY. Women's perception of helpful and unhelpful nursing behaviors during labor: a study in Taiwan. Birth 2001;28(3):180-185.

19. Harriott EM, Williams TV, Peterson MR. Childbearing in U.S. military hospitals: Dimensions of care affecting women's perceptions of quality and satisfaction. Birth 2005;32(1):4-10.

20. Eriksson C, Jansson L, Hamberg K. Women's experiences of intense fear related to childbirth investigated in a Swedish qualitative study. Midwifery 2006;22:240248.

21. Kornelsen J. Essences and imperatives: An investigation of technology in childbirth. Social Science and Medicine 2005;61(7):1495-1504.

22. Homer CSE, Davis GK, Cooke M, Barclay LM. Women's experiences of continuity of midwifery care in a randomised controlled trial in Australia. Midwifery 2002;18(2):102-112.

23. Kabakian-Khasholian T, Campbell O:S,M., Ghorayeb F. Women's experiences of maternity care: satisfaction or passivity. Social Science \& Medicine 2000;51:103113.

24. Green JM, Baston HA. Feeling in Control during Labor: Concepts, Correlates, and Consequences. Birth 2003;30(4):235-247.

25. Amooti-Kaguna B, Nuwaha F. Factors influencing choice of delivery sites in Rakai district of Uganda. Social Science and Medicine 2000;50(2):203-213.

26. Fenwick J, Hauck Y, Downie J, Butt J. The childbirth expectations of a self-selected cohort of Western Australian women. Midwifery, 2005;21: 23-35.

27. White PC. Crossing the river: Khmer women's perceptions of pregnancy and postpartum. Journal of Midwifery \& Women's Health 2002;47(4):239-246.

28. McCrea B, Wright ME. Satisfaction in childbirth and perceptions of personal control in pain relief during labor. Journal of Advanced Nursing, 199929(4), 877884.

29. Viisainen K. Negotiating control and meaning: Home birth as a self-constructed choice in Finland. Social Science and Medicine 2001;52(7):1109-1121.

30. Mozingo JN, Davis MW, Thomas SP, Droppleman PG. "I Felt Violated": Women's Experience of Childbirth-Associated Anger. MCN The American Journal of Maternal Child Nursing 2002;27(6):342-348.

31. Soto P. Gestión administrativa como soporte del proceso de enfermería. In: Soto P, García A, Ourcilleon A, Robles C, Vial B, Arratia A, editors. Apuntes de clases. 1st ed. Chile; 2004. p. 7-8. 